\title{
Bone marrow transplantation reveals an essential synergy between neuronal and hemopoietic cell neurokinin production in pulmonary inflammation
}

\author{
Mara Chavolla-Calderón, Meggan K. Bayer, and J. Julio Pérez Fontán \\ The Edward Mallinckrodt Department of Pediatrics, Washington University School of Medicine, St. Louis, Missouri, USA
}

\begin{abstract}
Neurogenic inflammation is believed to originate with the antidromic release of substance $P$, and of other neurokinins encoded by the preprotachykinin A $(P P T-A)$ gene, from unmyelinated nerve fibers (C-fibers) following noxious stimuli. Consistent with this concept, we show here that selective sensoryfiber denervation with capsaicin and targeted deletion of the PPT-A gene protect murine lungs against both immune complex-mediated and stretch-mediated injuries. Reconstitution of PPT- $A$ gene-deleted mice with WT bone marrow does not abrogate this protection, demonstrating a critical role for $P P T-A$ gene expression by sensory neurons in pulmonary inflammation. Surprisingly, reconstitution of WT mice with $P P T-A$ gene-deficient bone marrow also confers protection against pulmonary injury, revealing that $P P T-A$ gene expression in hemopoietic cells has a previously unanticipated essential role in tissue injury. Taken together, these findings demonstrate a critical synergy between capsaicin-sensitive sensory fibers and hemopoietic cells in neurokinin-mediated inflammation and suggest that such synergy may be the basis for a stereotypical mechanism of response to injury in the respiratory tract.

This article was published online in advance of the print edition. The date of publication is available

from the JCI website, http://www.jci.org. J. Clin. Invest. 111:973-980 (2003). doi:10.1172/JCI200317458.
\end{abstract}

\section{Introduction}

The observation more than 30 years ago that capsaicin stimulation of some afferent nerve fibers releases proinflammatory neuropeptides (1) led to the idea that injured or irritated sensory nerves can initiate inflammation or amplify the inflammatory responses initiated by noxious stimuli. Substance $P$ and the other neurokinins encoded by the preprotachykinin A (PPT-A) gene (2) play a fundamental role in this process, which is known as neurogenic inflammation. Neurokinins are thought to be stored primarily in unmyelinated nerve fibers (C-fibers) and released antidromically as part of the nociceptive response. The released molecules bind to a family of ubiquitous $G$ protein-coupled receptors with at least three distinct members, of which the neurokinin-1 receptor (NK-1R) has the highest affinity for substance P (3). Activation of NK-1R in smooth muscle cells, endothelial cells, and leukocytes decreases vascular tone, increases endothelial permeability, and promotes local inflow of inflammatory cells (4), thereby enhancing the inflammation induced by the original stimulus. Although

Received for publication November 22, 2002, and accepted in revised form February 4, 2003.

Address correspondence to: J. Julio Pérez Fontán, Department of Pediatrics, Washington University School of Medicine, St. Louis Children's Hospital, Suite 5S20, St. Louis, Missouri 63110, USA. Phone: (314) 454-2527; Fax: (314) 361-0733;

E-mail: fontan@kids.wustl.edu.

Conflict of interest: The authors have declared that no conflict of interest exists.

Nonstandard abbreviations used: preprotachykinin A (PPT-A); preprotachykinin B (PPT-B); neurokinin-1 receptor (NK-1R); macrophage inflammatory protein-1 $\alpha$ (MIP-1 $\alpha)$. increased substance $\mathrm{P}$ immunoreactivity has been found in bronchoalveolar lavage samples from humans suffering from lung diseases as diverse as asthma and the adult respiratory distress syndrome $(5,6)$, the true pathogenic potential of the PPT-A gene-encoded neurokinins in naturally occurring illness remains unknown.

Two recent observations, however, suggest that the prevalent understanding of neurogenic inflammation is in need of revision. First, the discovery that disruption of the $N K-1 R$ gene protects against the injury induced by antigen-antibody complex formation in the airways has revealed that neurokinin amplification serves an essential rather than a cooperative role, at least in this type of injury (7). In addition, the detection of PPT-A mRNAs and substance P-like immunoreactivity in intrinsic airway neurons $(8,9)$ and, more recently, in inflammatory cells (10-15) has uncovered the existence of two cellular reservoirs of neurokinins in the lungs, one constitutive and the other migrant. Questioned when first reported, the now confirmed presence of substance $P$ and other neurokinins in resident macrophages and circulating leukocytes is particularly intriguing because many of these cells also express neurokinin receptors $(10,14)$. Thus, it is possible that inflammatory cells use PPT-A gene-encoded neurokinins as a paracrine or autocrine signaling mechanism to propagate inflammation beyond the limited topographic spread of the C-fibers and intrinsic neurons (16).

Pursuant of this hypothesis, we report here that disruption of the PPT-A gene protects murine lungs against immune complex-mediated and mechanical stretch-mediated injuries. By applying an exclusionary strategy of selective capsaicin denervation and bone 
marrow reconstitution, we also show that the PPT-A gene must be functional in both sensory nerves and hemopoietic cells for these two types of injury to progress. Our findings demonstrate not only that $P P T-A$ gene-encoded neurokinins play a fundamental role in the amplification of two very distinct forms of inflammation in the lungs, but also that this role involves a previously unsuspected synergy between sensory nerve fibers and PPT-A gene-expressing inflammatory cells.

\section{Methods}

PPT-A gene-deficient mice. PPT-A gene-deficient mice were generated by nonsibling heterozygous crossings of mice carrying a targeted deletion of the substance $P$ and neurokinin A encoding regions of the PPT-A gene (these were a generous gift from A. Basbaum, University of California, San Francisco, San Francisco, California, USA) (17). Littermates lacking the mutant allele were used as matched WT controls for all the experiments. PPT-A gene-deficient mice cannot synthesize substance $\mathrm{P}$, neurokinin $\mathrm{A}$, and the neurokinin A extended peptides neuropeptide $K$ and neuropeptide $\gamma(2)$, leaving neurokinin $\mathrm{B}$, which is encoded by the PPT-B gene, as the only potential ligand for the neurokinin receptors. In preliminary studies, we ascertained that these mice have a normal TNF- $\alpha$ serum surge after the administration of endotoxin $(2.45 \pm 0.03 \mathrm{ng} / \mathrm{ml}$ in PPT-A gene-deleted mice vs. $2.4 \pm 0.02 \mathrm{ng} / \mathrm{ml}$ in WT mice 90 minutes after $10 \mathrm{mg}$ of LPS $/ \mathrm{kg}, n=3$, strain C57B/6J). We have also shown that macrophages isolated from the peritoneum of the same mice release TNF- $\alpha$ at concentrations commensurate with those of WT mice, independent of whether they were preincubated with substance $\mathrm{P}$ (data not shown).

The original line of PPT-A gene-deleted mice was both kept in a CD-1 background and backcrossed to inbred C57B/6J mice for eight or more generations. Experiments were performed separately in both strains to identify potential variability from gene epistasis. All animal care practices and experimental procedures were approved by the Washington University Institutional Animal Studies Committee.

Capsaicin-mediated sensory denervation. Selective denervation with capsaicin was used to define the contribution of neurokinins released by sensory fibers expressing the vanilloid TRPV1 receptor (18) to immune complex-mediated inflammation. Capsaicin causes release of substance $\mathrm{P}$ and other neurokinins from $\mathrm{C}$-fiber terminals, producing in the process a durable and extensive functional ablation of these fibers $(19,20)$, which is most complete when the capsaicin is given during the newborn period (21). The mice, CD-1 or C57B/6J, were injected with capsaicin (50 $\mathrm{mg} / \mathrm{kg}$ ) subcutaneously at day 2 or 3 after birth and studied at 12 weeks of age. At the time of the study, capsaicintreated mice exhibited normal behavior, and their circulating total and differential white cell counts $\left(11.9 \times 10^{3} \pm\right.$ $1.4 \times 10^{3}$ vs. $11.8 \times 10^{3} \pm 1.3 \times 10^{3}$ cells $/ \mu \mathrm{l}$, mean $\pm \mathrm{SE}$, $n=6)$, erythrocyte counts $\left(9.2 \times 10^{6} \pm 0.2 \times 10^{6}\right.$ vs. $\left.9.0 \times 10^{6} \pm 0.2 \times 10^{6}\right)$, and platelet counts $\left(693 \times 10^{3} \pm\right.$
$63 \times 10^{3}$ vs. $\left.498 \times 10^{3} \pm 9 \times 10^{3}\right)$ were similar to those of untreated mice of the same age and background.

Immune complex-mediated injury. The effects of immune complex-mediated injury were studied over a 4-hour period. Mice were anesthetized with $1.5 \%$ Avertin (1.5 ml/kg intraperitoneally), and their tracheae were cannulated through the mouth with a 22 -gauge Angiocath, using a pediatric otoscope to visualize the glottis. Chicken ovalbumin $(20 \mathrm{mg} / \mathrm{kg}$ in $0.5 \mathrm{ml} \mathrm{PBS}$; Sigma-Aldrich, St. Louis, Missouri, USA) was injected into the tail vein, and a polyclonal rabbit-raised antibody against ovalbumin $(10 \mathrm{mg} / \mathrm{kg}$ in $0.1 \mathrm{ml} \mathrm{PBS}$; Chemicon International Inc., Temecula, California, USA) was instilled into the trachea immediately thereafter (7). The endotracheal tube was removed, and the mouse was returned to its cage, with free access to water and chow. Each mouse was again anesthetized 20 minutes before the 4-hour end-point by isoflurane inhalation in a Plexiglas induction box, and Evans blue $(30 \mathrm{mg} / \mathrm{kg}$ in $0.1 \mathrm{ml}$ PBS; Sigma-Aldrich) was injected intravenously (22) to assess the effect of the immune complex-mediated inflammation on alveolar-capillary permeability. After administration of an overdose of $\mathrm{Na}$ pentobarbital, the chest and neck were opened by midline incision, and a blood sample was drawn from the right ventricle to determine the serum concentration of Evans blue. The trachea was cannulated by tracheostomy, and the left main-stem bronchus was ligated. The right lung was then lavaged with PBS in three passes of $1 \mathrm{ml}$ each. Finally, the left lung was removed and frozen for later analysis, and the right lung was fixated in situ with $1 \mathrm{ml}$ of $4 \%$ paraformaldehyde instilled into the trachea and removed for sectioning.

Mechanical ventilation. After cannulation of the trachea with a 22-gauge Angiocath under anesthesia with $\mathrm{Na}$ pentobarbital $(50 \mathrm{mg} / \mathrm{kg}$ intraperitoneally), the mouse was placed on the cradle of a small-rodent plethysmograph (Kent Scientific Corp., Litchfield, Connecticut, USA). The tracheal cannula was connected to the tubing of a Harvard rodent ventilator (Harvard Apparatus Inc., Holliston, Massachusetts, USA) using a Y-connector and tubing of minimal dead space. The ventilator was adjusted to provide an end-expiratory pressure of $2 \mathrm{~cm} \mathrm{H}_{2} \mathrm{O}$ and a tidal volume of either $6 \mathrm{ml} / \mathrm{kg}$ (peak airway pressure $9-10 \mathrm{~cm} \mathrm{H}_{2} \mathrm{O}$ ) or $20 \mathrm{ml} / \mathrm{kg}$ (peak airway pressure 16-17 $\mathrm{cm} \mathrm{H}_{2} \mathrm{O}$ ), using air as the ventilation gas. The breathing rate was adjusted to either 150 or 45 breaths per minute, depending on tidal volume, to maintain minute ventilation constant at $900 \mathrm{ml} /(\mathrm{kg} \times \mathrm{min})$. Preliminary experiments showed that these combinations of tidal volume and breathing rate maintained blood $\mathrm{pH}$ and $\mathrm{P}_{\mathrm{CO} 2}$ within physiological range. Next, the mouse was injected with $0.3 \mathrm{mg} / \mathrm{kg}$ of pancuronium bromide intraperitoneally for neuromuscular blockade. An additional dose of $25 \mathrm{mg} / \mathrm{kg}$ of $\mathrm{Na}$ pentobarbital was give 2 hours after the initial anesthetic dose or, at any point, if spontaneous movement was observed. Throughout the experiment, airway pressures and tidal volume were measured continuously using appropriate range differ- 
ential-pressure transducers (Validyne Engineering Corp., Northridge, California, USA). Signals for pressure and volume (derived from the time integral of the signal from the plethysmograph's calibrated-screen pneumotachometer) were displayed continuously and recorded on a computerized data-acquisition system (Biopac Systems Inc., Goleta, California, USA). At the end of a 4-hour period of mechanical ventilation, half of the mice were killed with an overdose of $\mathrm{Na}$ pentobarbital, and their lungs were lavaged with three passes of $1 \mathrm{ml}$ PBS each, fixated, and excised. The other half of the mice had their tracheal cannulae removed and were returned to their cages until 24 hours later, when they were killed with an overdose of $\mathrm{Na}$ pentobarbital and their lungs processed following a similar protocol. Alveolar-capillary permeability was determined by injection of Evans blue 20 minutes before the end of the experiments, as described above.

Bone marrow reconstitution experiments. To define the contribution of PPT-A gene-expressing hemopoietic cells to the amplification of both immune complex-mediated and stretch-mediated injuries, we performed bone marrow reconstitution experiments based on three permutations of donor and recipient: WT to WT mice, which served as controls for the effects of conditioning radiation and the bone marrow transplantation process; WT to PPT- $A$ gene-deleted mice, in which only bone marrow-derived cells gained the ability to synthesize PPT-A gene-encoded peptides; and PPT-A gene-deleted to WT mice, in which only bone marrow-derived cells lost the ability to synthesize PPT-A gene-encoded peptides. In the case of the CD-1 mice, littermates were used as donors; in the case of the C57BL/ 6 mice, pure-bred mice carrying a Ly-5 congenic marker were used as either WT donors or recipients to confirm the existence of complete chimerism in the circulating hemopoietic cells (23). In preparation, all recipient mice underwent conditioning with $1,000 \mathrm{cGy}$ from a ${ }^{137} \mathrm{Cs}$ source at a rate of $95 \mathrm{cGy} / \mathrm{min}$ the day before intravenous injection of the donor bone marrow. Femora from the donor mice were dissected and flushed with 1 $\mathrm{ml}$ Hebs (20 mM Hepes, $137 \mathrm{mM} \mathrm{NaCl}, 5 \mathrm{mM} \mathrm{KCl}, 0.7$ $\mathrm{mM} \mathrm{Na}_{2} \mathrm{HPO}_{4}, 6 \mathrm{mM}$ dextrose). The resultant cell suspension was centrifuged at $400 \mathrm{~g}$ and resuspended in 10 $\mathrm{ml}$ PBS. The supernatant was centrifuged again at $350 \mathrm{~g}$, and the pellet was resuspended in $1 \mathrm{ml}$ PBS. Cells were counted and diluted to administer a total dose of $2 \times 10^{6}$ nucleated cells. A wait period of at least 145 days was allowed for bone marrow engraftment and for the turnover of any hemopoietic cells that survived the conditioning irradiation. Previous studies have shown that, while other bone marrow-derived cell compartments are repopulated quickly, lung macrophages may require up to 90 days to achieve complete chimerism after bone marrow transplantation (24).

Assessment of inflammatory injury and detection of substance P. Cells in the bronchoalveolar lavage fluid were counted in a hemocytometer after centrifugation (at $63 \mathrm{~g}$ for 5 minutes) in an automatic slide-plating system (Cytofunnel; Thermo Shandon Inc., Pittsburgh, Penn- sylvania, USA), fixation, and staining with eosin and methylene blue. Evans blue concentrations in serum and bronchoalveolar lavage fluid were determined spectrophotometrically at $620 \mathrm{~nm}$. Casein zymograms were performed as described previously to obtain a semiquantitative assessment of protease activity (9). Substance P, TNF- $\alpha$, and macrophage inflammatory protein-1 $\alpha$ (MIP-1 $\alpha)$ concentrations in the bronchoalveolar lavage fluid were measured by duplicates with commercial ELISA kits (Cayman Chemical Co., Ann Arbor, Michigan, USA, for substance P; Quantikine M from R\&D Systems Inc., Minneapolis, Minnesota, USA, for TNF- $\alpha$ and MIP-1 $\alpha$ ). Immunostaining for substance $P$ was performed in paraffin-embedded sections using a guinea pig polyclonal primary antibody (Chemicon International Inc.) at 1:200 dilution and alkaline phosphatase immunohistochemistry (Vector Laboratories Inc., Burlingame, California, USA) for detection.

Differences between groups were tested by ANOVA or by the Kruskal-Wallis test, depending on whether the data followed a normal distribution. Tissues were cut in $10-\mu \mathrm{m}$ sections and stained with $\mathrm{H} \& \mathrm{E}$ according to standard technique. For the ventilation studies, where the injury was less severe and differences in tissue inflammation more difficult to discern, we asked a colleague uninvolved in the work to rate blindly two equal random samples ( $n=6$ per group) of tissue sections from WT and PPT-A gene-deleted mice. A score of 0-2 was assigned to the inflammatory response observed in each slide $(0$, no inflammation; 1 , mild infiltration with neutrophils or hemorrhage; and 2 , severe infiltration with neutrophils or hemorrhage). The scores were compared by $\chi^{2}$ analysis.

\section{Results}

Effect of PPT-A gene deletion and capsaicin-selective denervation on immune complex-mediated inflammation. Simultaneous intravenous injection of chicken ovalbumin and intratracheal instillation of a polyclonal antibody against chicken ovalbumin produced a marked inflammatory response in the untreated WT mice, which by 4 hours was characterized by hemorrhage, exudation of neutrophils, increased passage of Evans blue, and release of TNF- $\alpha$ and proteases into the alveoli (Figure 1). This response was markedly attenuated in the mice with the deleted PPT-A gene and in the mice treated with capsaicin at birth. The effects of PPT-A gene deletion and capsaicin denervation were similar in CD-1 and $\mathrm{C} 57 \mathrm{~B} / 6 \mathrm{~J}$ mice; the results obtained in these two strains are therefore reported together.

Effect of PPT-A gene deletion on stretch-mediated inflammation. At the end of the 4-hour period of mechanical ventilation, there were no detectable differences in the bronchoalveolar lavage fluid or the lung tissues obtained from the WT and PPT-A gene-deleted mice, except for a small, yet significant, elevation in the TNF- $\alpha$ levels in the bronchoalveolar lavage fluid from the WT mice ventilated with high tidal volumes (Figure 2). When studied 24 hours later, however, WT mice whose 


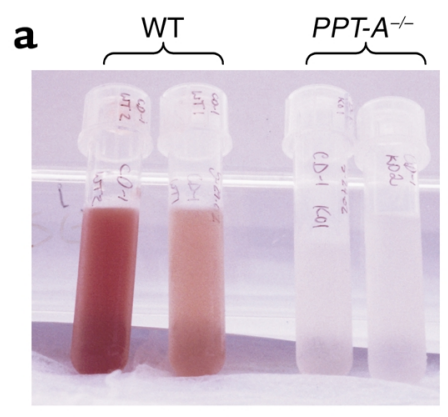

\section{b}

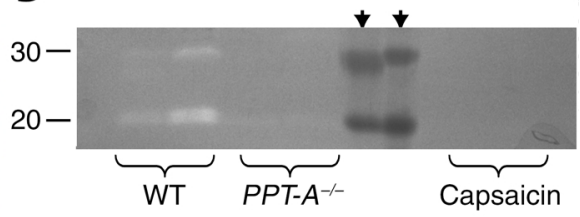

C
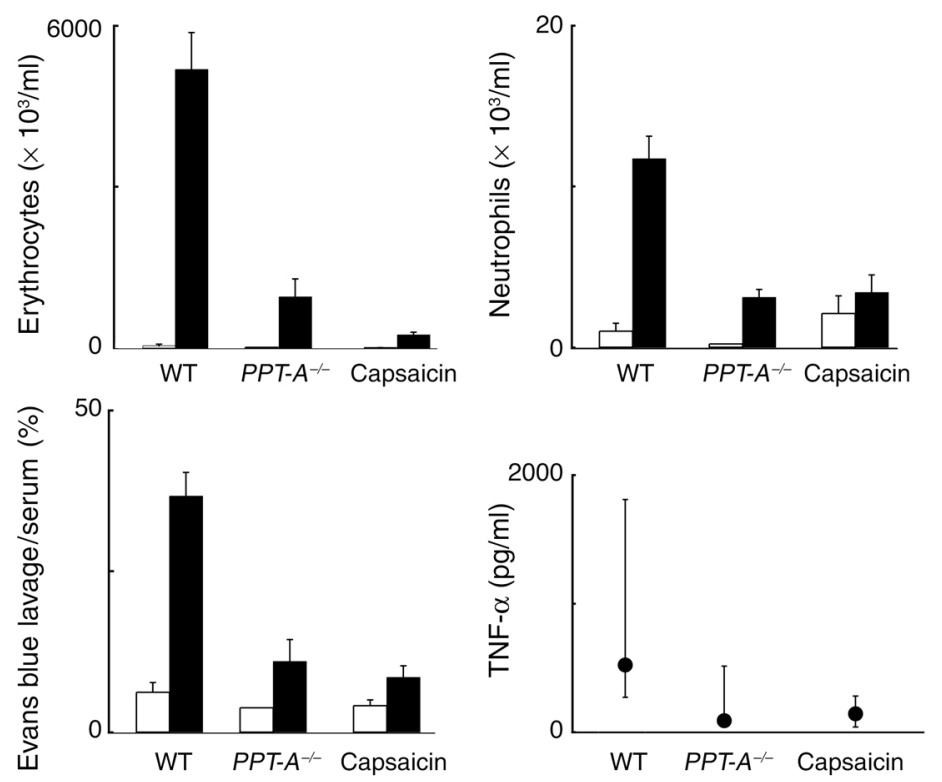

\section{Figure 1}

Attenuation of immune complex-mediated injury by PPT- $A$ gene deletion and capsaicin-selective sensory denervation. (a) Bronchoalveolar lavage fluid obtained after intravenous injection of chicken ovalbumin and intratracheal instillation of a polyclonal antibody against ovalbumin shows alveolar hemorrhage in WT mice, but not in mice with targeted deletion of the PPT-A gene $\left(P P T-A^{-/-}\right)$. (b) Casein zymogram demonstrates increased bronchoalveolar lavage fluid protease activity in WT compared with $P P T-A^{-/-}$and capsaicin-denervated mice. The proteolytic bands visible in the illustration have the electrophoretic mobility profiles of matrilysin and macrophage metalloelastase. Black arrowheads indicate molecular weight markers. (c) Erythrocyte and neutrophil counts in the bronchoalveolar lavage fluid, Evans blue lavage/serum concentration ratios, and TNF- $\alpha$ levels in the bronchoalveolar lavage fluid demonstrate protection against immune complex injury by PPT-A gene deletion and capsaicin denervation $(P<0.0001$, except for TNF- $\alpha$ levels where $P=0.01$ ). Cell counts and Evans blue ratios (black bars; $n=18 \mathrm{WT}, 11 P P T-A^{-/}$, and 19 capsaicin) are compared with those of control mice injected intravenously with ovalbumin and intratracheally with normal saline (white bars; $n=8 \mathrm{WT}, 2$ PPT- $A^{-/-}$, and 12 capsaicin). TNF- $\alpha$ levels were measured in fewer mice ( $n=10 \mathrm{WT}, 7$ PPT- $A^{-/-}$, and 11 capsaicin). Values are shown as mean \pm SE, except for TNF- $\alpha$ levels, which are shown as median and 25 th to 75 th percentile span. lungs had been ventilated with high tidal volumes developed an injury characterized by inflammatory infiltration of the lung interstitium and alveolar hemorrhage, exudation of neutrophils and macrophages into the alveoli, increased alveolar-capillary permeability, and elevated levels of TNF- $\alpha$ and MIP- $1 \alpha$ in the bronchoalveolar lavage fluid (Figure 2). Mice with deleted PPT-A genes were protected against this injury, as shown both by blinded morphologic assessment of tissue cuts (median injury score of 0 vs. $1, \chi^{2}, P=0.014$ ) and by analysis of the bronchoalveolar lavage fluid (Figure 2).

Contribution of PPT-A gene-expressing hemopoietic cells to immune complex-mediated and stretch-mediated inflammation. WT mice whose bone marrow had been reconstituted with WT bone marrow and nontransplanted WT mice exhibited a similar injury after immune complex formation (Figure 3). In contrast, mice with PPT-A gene deletions retained protection against immune complex-mediated injury after their bone marrow was reconstituted with WT bone marrow, confirming that PPT-A gene-expressing hemopoietic cells are not by themselves sufficient for this injury. Surprisingly, WT mice gained protection against the injury after their bone marrow was reconstituted with PPT-A-deficient cells, indicating that PPT-A gene expression in hemopoietic cells is essential for immune complex-mediated inflammation in the lungs. Immunostaining of the lung tissues after both immune complex-mediated (Figure 4) and stretch-mediated injuries revealed substance P immunoreactivity only in alveolar and tissue macrophages in WT mice, and in both WT and PPT-A gene-deleted mice reconstituted with WT bone marrow. In the bronchoalveolar lavage fluid, substance P immunoreactivity was decreased by $70-80 \%$ both in PPT-A gene-deleted mice reconstituted with WT bone marrow and in WT mice reconstituted with PPT-A gene-deleted bone marrow (Figure 4). We found no difference in the responses from CD-1 and C57B/6J mice, and therefore the results from both strains are reported together.

Because of the speed and severity with which inflammation develops after immune complex formation, we decided to focus our analysis of the contributions of capsaicin-sensitive neurons and PPT-A gene-expressing hemopoietic cells primarily on this type of injury. To determine, however, whether the ensuing conclusions would also apply to stretch-mediated injury, we replicated the capsaicin and bone marrow reconstitution paradigms on a smaller sample of $\mathrm{C} 57 \mathrm{~B} / 6 \mathrm{~J}$ mice. The mice were ventilated for 4 hours with a high tidal volume and studied only after 24 hours, making the potential injury most detectable. Capsaicin-treated mice, PPT-A genedeleted mice reconstituted with WT bone marrow, and WT mice reconstituted with PPT-A gene-deleted cells all exhibited substantial attenuation of their inflammatory 
injuries compared with control WT mice reconstituted with WT bone marrow (Figure 5).

\section{Discussion}

Our results demonstrate that PPT-A gene-encoded neurokinins are essential for the lung injuries produced by two very diverse mechanisms: immune complex formation and mechanical stretch. We also show that progression of the inflammation produced by both types of injury requires concomitant expression of the PPT-A gene by capsaicin-sensitive sensory neurons and hemopoietic cells. Such a requirement is unprecedented, because it implies that nerves and inflammatory cells not only cooperate to amplify or propagate inflammation but also depend on a singular neuropeptidergic signaling system for their cooperation. Neurokinins appear to intervene very proximally in the ensuing inflammatory cascade, as denoted by the absence of detectable elevations in TNF- $\alpha$ concentrations in the bronchoalveolar lavage fluid of the capsaicin-treated and PPT- $A$ gene-deleted mice after immune complex formation, as well as by the absence of similar elevations in TNF- $\alpha$ and MIP- $1 \alpha$ concentrations in the PPT-A gene-deleted mice after mechanical ventilation.
Although its scale is surprising, the protection conferred by PPT-A gene deletion against immune complex-mediated injury is consistent with the findings previously reported in mice with disrupted $N K-1 R$ genes (7). Of the various neurokinins encoded by the PPT-A gene, substance $P$ exhibits the highest affinity for this receptor, and therefore it is likely that its suppression contributed substantively to attenuation of the inflammatory response. However, unlike NK-1R disruption (7), PPT-A gene deletion also abrogated the TNF- $\alpha$ surge found in the WT mice 4 hours after the immune complex injury, suggesting that substance $P$ or, more likely, neurokinin A induces cytokine release via neurokinin receptors other than NK-1R.

Stretch-mediated inflammation has been increasingly recognized as a key mechanism of injury during mechanical ventilation $(25,26)$ and is one of the reasons why low tidal volumes have recently been recommended for patients with acute lung injury (27). Our findings demonstrate, for the first time to our knowledge, that the inflammation produced by stretch can progress beyond the duration of the stimulus and that this progression requires the participation of PPT-A gene-encoded neurokinins. The alveolar-capillary injury, as ascer- $\mathbf{a}$

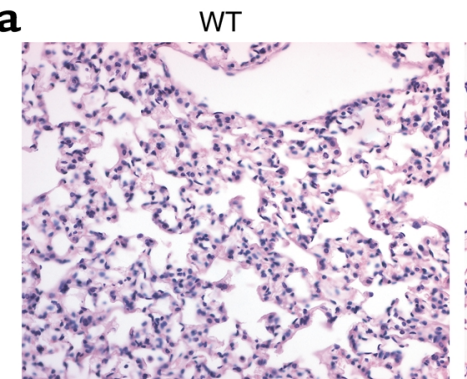

C

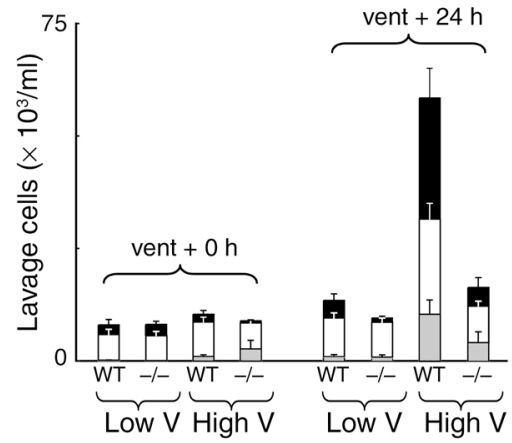

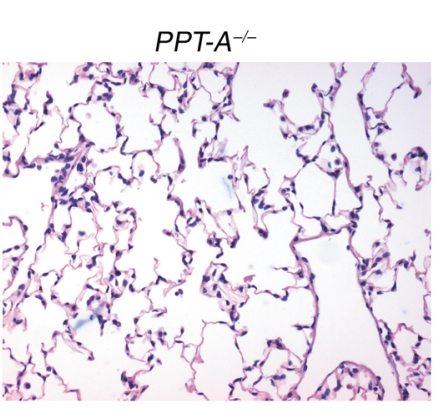

d

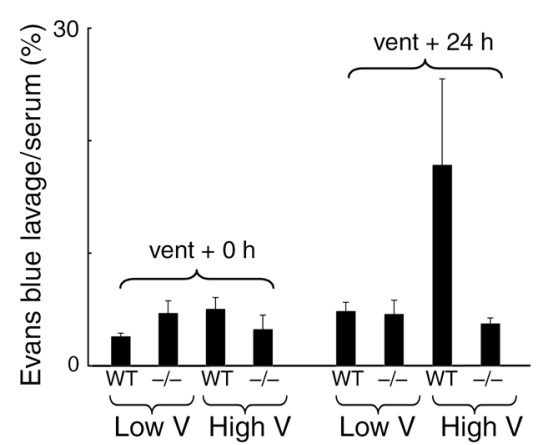

b

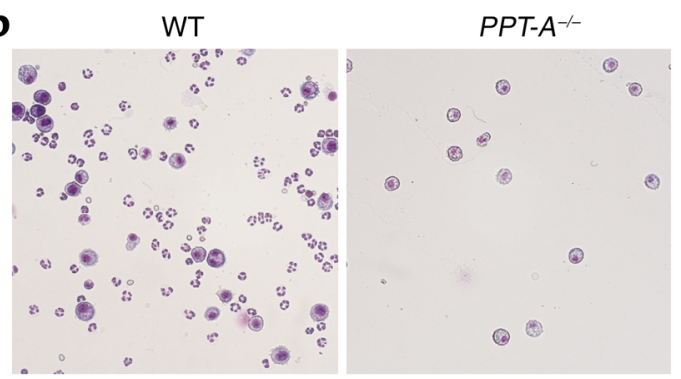

e

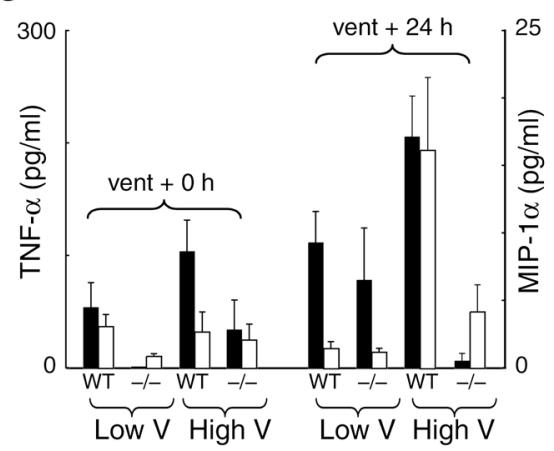

Figure 2

Protection conferred against stretch-mediated injury by PPT-A gene deletion. (a) H\&E-stained sections ( $\times 20)$ obtained 24 hours after a 4-hour period of mechanical ventilation with a tidal volume of $20 \mathrm{ml} / \mathrm{kg}$ demonstrate edema and inflammatory infiltration of the pulmonary interstitium in WT mouse but not in PPT-A gene-deleted $\left(P P T-A^{-/-}\right)$mouse. (b) Microphotograph $(\times 40)$ of bronchoalveolar lavage fluid after similar treatment shows exudation of macrophages and neutrophils in WT mouse, but only small numbers of macrophages in $P P T-A^{-/-}$mouse. (c) Cell counts in the bronchoalveolar lavage fluid ( $n=10$ for each combination of variables) show increased numbers of neutrophils (gray bars), macrophages (white bars), and erythrocytes (black bars) in WT but not in PPT-A $A^{-/}$mice 24 hours after ventilation (vent $+24 \mathrm{~h}$ ) with high tidal volumes (High V) $(P<0.0001)$. (d) Evans blue lavage/serum ratios $(n=10)$ demonstrate increased alveolar-capillary permeability in WT but not in $P P T-A^{-/-}$mice 24 hours after High V $(P<0.002)$. (e) TNF- $\alpha$ levels (black bars; $\left.n=5\right)$ were higher in WT than in $P P T-A^{-/-}$mice after ventilation with High $\mathrm{V}$, both at the end of the 4-hour ventilation period (vent $+0 \mathrm{~h} ; P=0.02)$ and 24 hours later $(P<0.0001)$. MIP-1 $\alpha$ levels $($ white bars; $n=5)$ were higher in WT than in PPT- $A^{-/-}$mice after High $\mathrm{V}$, but only after 24 hours $(P<0.0001)$. Values are shown as mean $\pm \mathrm{SE}$. 

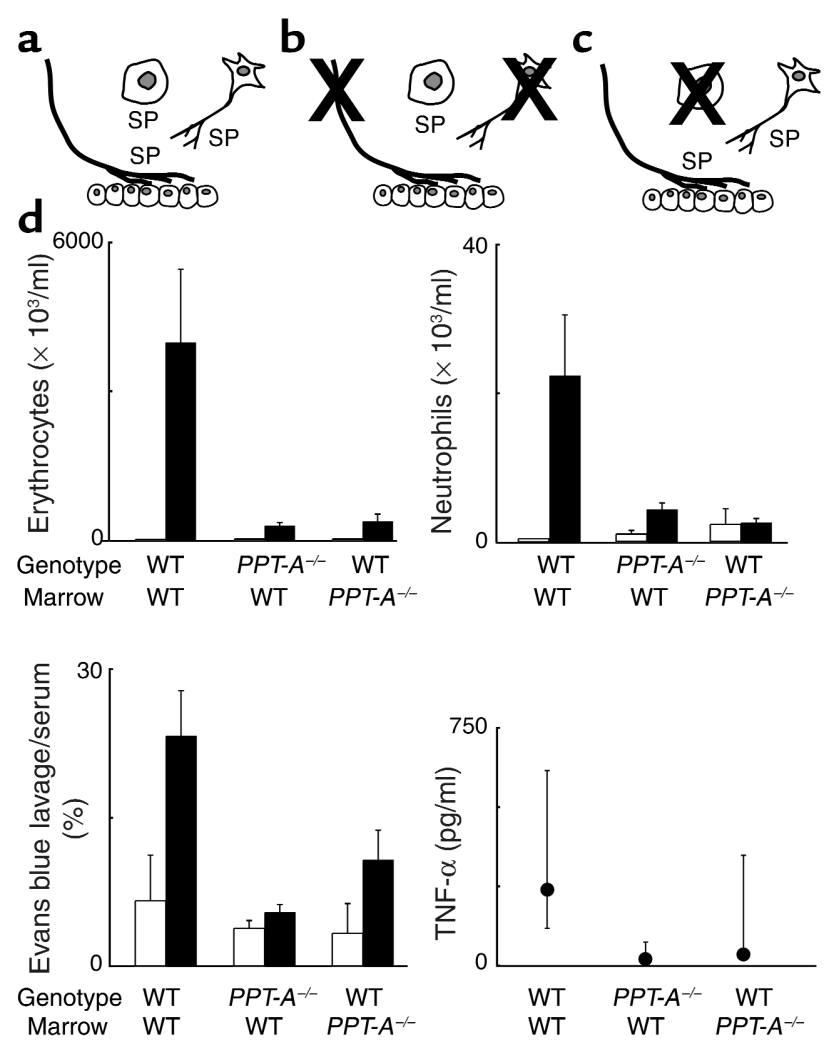

Figure 3

Effects of bone marrow reconstitution with WT cells in WT and PPT-A gene-deleted (PPT- $\left.A^{-/-}\right)$mice and with PPT- $A^{-/-}$cells in WT mice on immune complex-mediated lung injury. (a) WT mice reconstituted with WT bone marrow after conditioning irradiation. (b) PPT-A $A^{-/-}$mice (shown by crossing of cells affected by the gene deletion) reconstituted with WT bone marrow, restoring the ability of their hemopoietic cells to produce substance P (SP) and other $P P T-A$ gene-encoded neurokinins. (c) WT mice reconstituted with $P P T-A^{-/-}$bone marrow, eliminating the ability of their hemopoietic cells to produce PPT-A gene-encoded neurokinins. (d) WT mice reconstituted with WT bone marrow $(n=12)$ developed intense inflammation after immune complex formation. Reconstitution of $P P T-A^{-/-}$mice with WT bone marrow did not reestablish the inflammatory response $(n=15 ; P<0.0001)$. Reconstitution of WT mice with $P P T-A^{-/-}$bone marrow protected against this response $(n=15$; $P<0.0001)$. Cell counts and Evans blue ratios are shown in comparison with control mice injected intravenously with ovalbumin and intratracheally with normal saline (white bars; $n=4 \mathrm{WT}$ bone marrow to $\mathrm{WT}, 6 \mathrm{PPT}-\mathrm{A}^{-/}$- bone marrow to $\mathrm{WT}$, and $3 \mathrm{WT}$ bone marrow to $P P T-A^{-/-}$mice). TNF- $\alpha$ levels were determined in fewer mice $(n=7,10$, and 12 , respectively). Values are shown as mean $\pm \mathrm{SE}$, except for TNF- $\alpha$ levels, which are shown as median and 25 th to 75th percentile span.

tained by the bronchoalveolar lavage/serum Evans blue concentration ratio, nearly quadrupled in the 24 hours after discontinuation of mechanical ventilation. The fact that the vast increase in alveolar-capillary permeability implied by this finding is neurokinin-dependent detracts from the common view that the majority of the damage produced by lung stretch results from mechanical disruption (26), and it raises the possibility of new selective pharmacological therapies in the prevention of ventila- tor-induced lung injury. Moreover, the sustained suppression of inflammation in the PPT- $A$ gene-deficient mice suggests that the neurokinin role in inflammatory amplification is not supplanted over time by other cytokine signaling pathways.

After their initial discovery in activated peritoneal macrophages (28), the presence of PPT-A mRNAs and peptides in hemopoietic cells has been the subject of considerable curiosity. The results of the bone marrow reconstitution experiments reported here demonstrate that expression of the PPT-A gene in these cells is indeed crucial for the full development of both immune complex-mediated and stretch-mediated injury in the lungs. At this time, we cannot ascertain with complete certainty which specific hemopoietic cell lineage or lineages are involved in these injuries to the exclusion of other cell lines. The candidate cells must express the PPT- $A$ gene, be radiosensitive or otherwise have a short life cycle, and depend on bone marrow progenitors for regeneration. Macrophages fulfill all these conditions. Consistent with the findings of other investigators $(11,13)$, our immunohistochemical analysis showed that alveolar and tissue macrophages, but not neutrophils (the other hemopoietic cell found in abundance in the injured lung's interstitium and alveolar spaces), were immunoreactive for substance P. Resident macrophages have a clear topographic advantage to instigate inflammatory events because of their proximity to the injured epithelium (and, by extension, to sensory nerve fibers that contain substance $\mathrm{P})(13,29)$. As an important note of caution, however, neurokinins are usually synthesized in low copy numbers and may be difficult to detect by immunological methods. Thus, only a systematic exclusionary analysis of all airway cells of hemopoietic lineage may identify with any certainty the cell line or lines involved in the neurokinin amplification mechanism revealed by our study.

Taken together, the results of the capsaicin and bone marrow reconstitution studies provide important insights into the mechanisms by which sensory neurons and PPT-A gene-expressing hemopoietic cells may cooperate in the amplification of the inflammatory response. First, the protection conferred by PPT- $A$ gene deletion against immune complex-mediated and stretch-mediated injuries indicates that epithelial cells, even if they are almost certain to have released proinflammatory cytokines, cannot by themselves induce a full inflammatory response. Second, the fact that capsaicin pretreatment produced a level of protection similar to that conferred by PPT-A gene deletion confirms the long-held notion that sensory fibers that express vanilloid receptors $(19,20)$ are essential for neurogenic inflammation. To our knowledge, only this subset of nerve cells have been shown to contain neurotoxic variants of the vanilloid receptor, and therefore it is highly unlikely that the effects of capsaicin given during the newborn period abrogated neurokinin production by other cell lines 12 weeks later. Finally, the failure of WT bone marrow reconstitution of PPT-A gene-deleted 

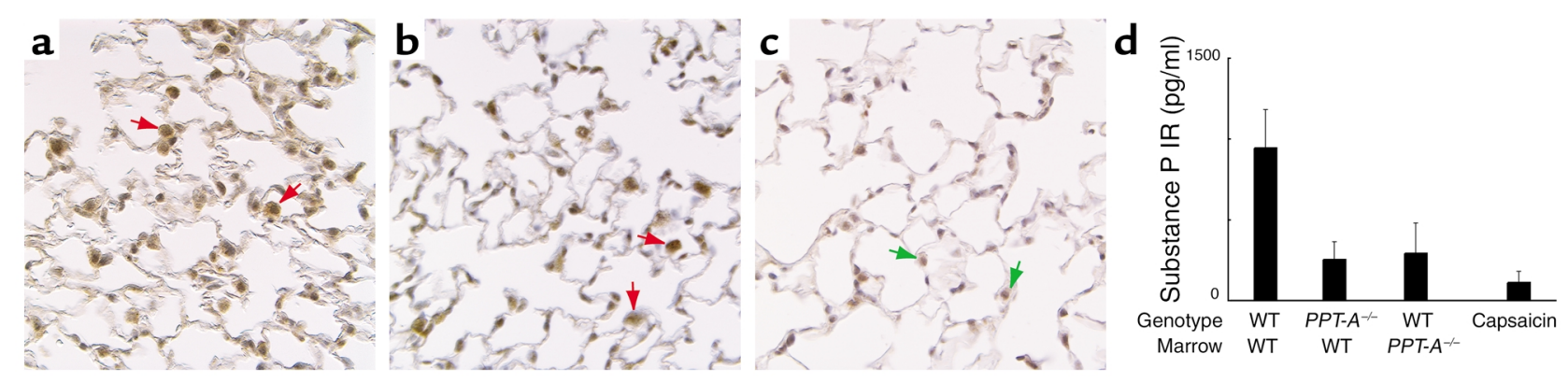

\section{Figure 4}

(a-c) Immunostaining of lung tissue for substance P after immune complex-mediated injury. Substance P immunoreactivity (IR, red arrows) was detected only in macrophages (but not other inflammatory cells) of WT mice reconstituted with WT bone marrow (a) and PPT- $A$ gene-deleted mice reconstituted with WT bone marrow $(\mathbf{b})$. No substance $\mathrm{P}$ immunoreactivity was present in macrophages (green arrows) or other cells in PPT-A gene-deleted mice (c). (d) Decreased substance P immunoreactivity (IR), by ELISA, in the bronchoalveolar fluid of WT, PPT-A gene-deleted $\left(P P T-A^{-/-}\right)$mice reconstituted with WT bone marrow, WT mice reconstituted with $P P T-\mathrm{A}^{-/-}$bone marrow, and capsaicinpretreated mice, after an immune complex-mediated lung injury $(P=0.03)$. Comparisons are relative to WT mice reconstituted with WT bone marrow, which serve as a WT control for the effects of conditioning irradiation. Values are shown as mean $\pm \mathrm{SE}(n=5$ for all groups).

mice to restore susceptibility to both immune complex-mediated and stretch-mediated injury, and the more surprising protection conferred by PPT-A gene-deleted bone marrow reconstitution of WT mice, demonstrate that neither sensory neurons nor PPT-A gene-expressing hemopoietic cells are by themselves sufficient to induce a full injury.

What, then, is the nature of the synergy between sensory neurons and neurokinin-producing hemopoietic cells? One potential answer is that suppression of the PPT-A gene simply renders inflammatory cells less reactive, as has been suggested for peritoneal macrophages exposed to bacterial endotoxin (30). A paracrine priming effect of this nature is unlikely to have contributed to our findings, however, because PPT-A gene-deleted hemopoietic cells were exposed to other PPT-A gene-competent cells from the time of their engraftment. Autocrine priming is also unlikely to be an important contributor, because WT hemopoietic cells failed to abrogate the protection of PPT-A gene-deleted mice against immune complex-mediated inflammation. These mice exhibit no evidence of neurokinin receptor downregulation in NK-1R-expressing cells (17) and, as noted above (see Methods), appear to have normal in vivo and in vitro cytokine responses to endotoxin. The fact that NK-1R disruption also abolishes

\footnotetext{
Figure 5

Protection conferred by reconstitution of WT mice with PPT-A gene-deleted $\left(P P T-A^{-/-}\right)$bone marrow and by capsaicin-selective denervation against inflammation (neutrophil counts in bronchoalveolar fluid; $P=0.0005$ ) and increased alveolar-capillary permeability (Evans blue lavage fluid/serum ratio; $P=0.0014$ ) 24 hours after a 4-hour period of high-volume ventilation. These results demonstrate, in a manner similar to that shown for immune complex-mediated inflammation (see Figure 3 ), that PPT-A gene expression by hemopoietic cells is critical for the progression of stretch-mediated inflammation. Values are shown as mean $\pm \mathrm{SE}(n=5$ for WT-to-WT reconstitution and 4 for all other groups).
}

the inflammation produced by immune complexes (7) may provide a hint to resolve the nature of the synergy between sensory fibers and hemopoietic cells. Because C-fibers do not express neurokinin receptors, but inflammatory cells do, it seems reasonable to think that neurokinins produced by subepithelial sensory neurons are needed to recruit neutrophils, macrophages, and possibly other cells such as mast cells into the inflammatory process, rather than the contrary. Inflammatory cells then may use substance $P$ as an autocrine or paracrine signal, thereby amplifying and propagating the inflammatory response. This interpretation is consistent with the virtual absence of

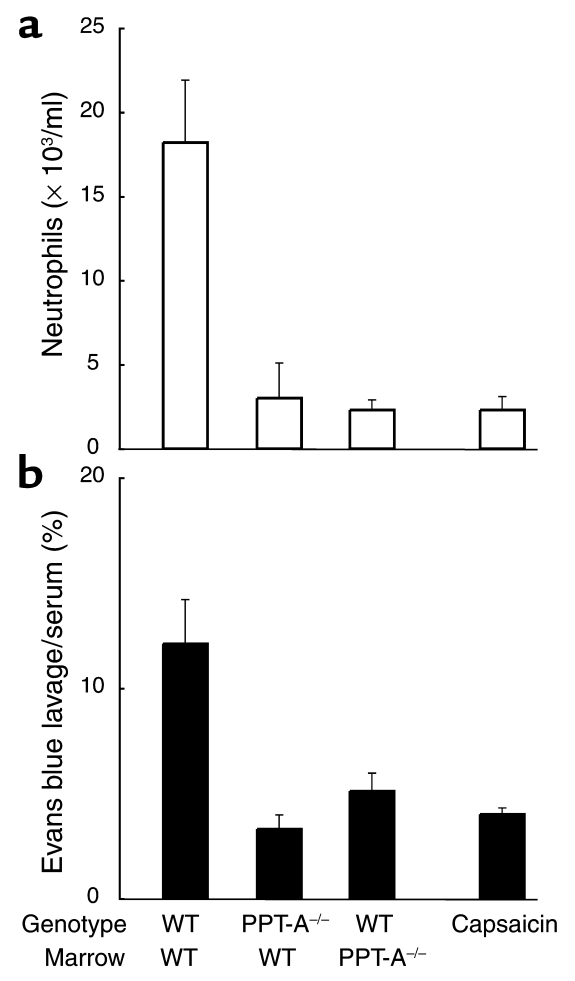


substance $\mathrm{P}$ immunoreactivity in the capsaicin-treated mice, and with the reduced but detectable immunoreactivity levels found in WT mice after reconstitution with PPT-A gene-deleted cells. The amount of neurokinins produced by sensory neurons may simply be insufficient to generate an inflammatory response in the absence of another neurokinin source. Alternatively, it is possible that the limiting factor is not quantity, but the reduced spread of these fibers - which are rare in the distal portions of the acinus where the immune complex-mediated and stretch-mediated injuries appear to have been most severe $(19,20)$ - or their inability to sustain neurokinin release through a process that requires transport of newly synthesized peptides from the cell bodies.

The findings reported here demonstrate that, far from serving as a vestigial system of nociception, PPT-A gene-encoded neurokinins play an essential role in the progression of very diverse inflammatory injuries. The requirement that $P P T-A$ gene-encoded peptides are produced by both vanilloid receptor-bearing afferents and hemopoietic cells suggests that substance $P$ or its related peptides are part of a stereotypical response to inflammatory stimuli in the respiratory tract. This response may well start by an interaction between injured airway epithelial cells and substance $\mathrm{P}$-containing subepithelial C-fibers and evolve through an autocrine or paracrine system of neuropeptidergic amplification by inflammatory cells. An interaction between nerves and hemopoietic cells similar to the one described here may be important in other systems where the participation of neurokinins in the genesis of acute inflammation is beginning to be recognized $(31,32)$. Pharmacological blockade of the neurokinin receptors may in that case offer a promising therapeutic option in the control of multiple forms of inflammatory injury.

\section{Acknowledgments}

This work was supported by grant HL-57998 from the National Heart, Lung, and Blood Institute. M. Chavolla-Calderón is the recipient of a Fellowship Award from the American Heart Association. We thank Timothy Graubert for his advice on bone marrow transplantation procedures, John K. McGuire for evaluating lung tissues for inflammation, Betsy Boedeker and Melinda Garrett for their technical assistance, and Alan Schwartz, Jonathan Gitlin, and Bill Parks for their critical review of the manuscript.

1. Jancsó, N., Jancsó-Gábor, A., and Szolcsányi, J. 1967. Direct evidence for neurogenic inflammation and its prevention by denervation and by pretreatment with capsaicin. Br. J. Pharmacol. 31:138-151.

2. Carter, M.S., and Krause, J.E. 1990. Structure, expression, and some regulatory mechanisms of the rat preprotachykinin gene encoding substance P, neurokinin A, neuropeptide K, and neuropeptide $\gamma$. J. Neurosci. 10:2203-2214.

3. Helke, C.J., Krause, J.E., Mantyh, P.W., Couture, R., and Bannon, M.J. 1990. Diversity in mammalian tachykinin peptidergic neurons: multiple peptides, receptors, and regulatory mechanisms. FASEB J. 4:1606-1615.

4. McDonald, D.M., Bowden, J.J., Baluk, P., and Bunnett, N.W. 1996. Neurogenic inflammation. A model for studying efferent actions of sensory nerves. Adv. Exp. Med. Biol. 410:453-462.
5. Espiritu, R.F., Pittet, J.F., Matthay, M.A., and Goetzl, E.J. 1992. Neuropeptides in pulmonary edema fluid of adult respiratory distress syndrome. Inflammation. 16:509-517.

6. Tomaki, M., et al. 1995. Elevated substance P content in induced sputum from patients with asthma and patients with chronic bronchitis. Am. J. Respir. Crit. Care Med. 151:613-617.

7. Bozic, C.R., Lu, B., Höpken, U.E., Gerard, C., and Gerard, N.P. 1996. Neurogenic amplification of immune complex inflammation. Science. 273:1722-1725.

8. Dey, R.D., Altemus, J.B., and Michalkiewicz, M. 1991. Distribution of vasoactive intestinal peptide- and substance P-containing nerves originating from neurons of airway ganglia in cat bronchi. J. Comp. Neurol. 304:330-340.

9. Pérez Fontán, J.J., et al. 2000. Substance P and neurokinin-1 receptor expression by intrinsic airway neurons in the rat. Am. J. Physiol. 278:L344-L355.

10. Ho, W.Z., Lai, J.P., Zhu, X.H., Uvaydova, M., and Douglas, S.D. 1997. Human monocytes and macrophages express substance $P$ and neurokinin-1 receptor. J. Immunol. 159:5654-5660.

11. Killingsworth, C.R., Shore, S.A., Alessandrini, F., Dey, R.D., and Paulauskis, J.D. 1997. Rat alveolar macrophages express preprotachykinin gene-I mRNA-encoding tachykinins. Am. J. Physiol. 273:L1073-L1081.

12. Pascual, D.W., and Bost, K.L. 1990. Substance P production by P388D1 macrophages: a possible autocrine function for the neuropeptide. Immunology. 71:52-56.

13. Castagliuolo, I., et al. 1997. Increased substance P responses in dorsal root ganglia and intestinal macrophages during Clostridium difficile toxin A enteritis in rats. Proc. Natl. Acad. Sci. U. S. A. 94:4788-4793.

14. Lai, J.P., Douglas, S.D., and Ho, W.Z. 1998. Human lymphocytes express substance P and its receptor. J. Neuroimmunol. 86:80-86.

15. Metwali, A., et al. 1994. Eosinophils within the healthy or inflamed human intestine produce substance $P$ and vasoactive intestinal peptide. J. Neuroimmunol. 52:69-78.

16. Coleridge, J.C.G., and Coleridge, H.M. 1984. Afferent vagal C fibre innervation of the lungs and airways and its functional significance. Rev. Physiol. Biochem. Pharmacol. 99:2-110.

17. Cao, Y.Q., et al. 1998. Primary afferent tachykinins are required to experience moderate to intense pain. Nature. 392:390-393.

18. Caterina, M.J., et al. 2000. Impaired nociception and pain sensation in mice lacking the capsaicin receptor. Science. 288:306-313.

19. Lundberg, J.M., Brodin, E., and Saria, A. 1983. Effects and distribution of vagal capsaicin-sensitive substance $P$ neurons with special reference to the trachea and lungs. Acta Physiol. Scand. 119:243-252.

20. Lundberg, J.M., Hokfelt, T., Martling, C.-R., Saria, A., and Cuello, C. 1984. Substance-P-immunoreactive sensory nerves in the lower respiratory tract of various mammals including man. Cell Tissue Res. 235:251-261.

21. Nagy, J.I., Hunt, S.P., Iversen, L.L., and Emson, P.C. 1981. Biochemical and anatomical observations on the degeneration of peptide-containing primary afferent neurons after neonatal capsaicin. Neuroscience. 6:1923-1934.

22. Patterson, C.E., Rhoades, R.A., and Garcia, J.G.N. 1992. Evans blue dye as a marker of albumin clearance in cultured endothelial monolayer and isolated lung. J. Appl. Physiol. 72:865-873.

23. Kawada, H., and Ogawa, M. 2001. Bone marrow origin of hematopoietic progenitors and stem cells in murine muscle. Blood. 98:2008-2013.

24. Nakata, K., et al. 1999. Augmented proliferation of human alveolar macrophages after allogeneic bone marrow transplantation. Blood. 93:667-673.

25. Tremblay, L., Valenza, F., Ribeiro, S.P., Li, J., and Slutsky, A.S. 1997. Injurious ventilatory strategies increase cytokines and c-fos mRNA expression in an isolated rat lung model. J. Clin. Invest. 99:944-952.

26. Dreyfuss, D., and Saumon, G. 1998. Ventilator-induced lung injury: lessons from experimental studies. Am. J. Respir. Crit. Care Med. 157:294-323.

27. 2000. Ventilation with lower tidal volumes as compared with traditional tidal volumes for acute lung injury and the acute respiratory distress syndrome. The Acute Respiratory Distress Syndrome Network. N. Engl. J. Med. 342:1301-1308.

28. Bost, K.L., and Pascual, D.W. 1992. Substance P: a late-acting B lymphocyte differentiation cofactor. Am. J. Physiol. 262:C537-C545.

29. Lotz, M., Vaughan, J.H., and Crason, D.A. 1988. Effect of neuropeptides on production of inflammatory cytokines by human monocytes. Science. 241:1218-1220

30. Berman, A.S., Chancellor-Freeland, C., Zhu, G., and Black, P.H. 1996. Substance $P$ primes murine peritoneal macrophages for an augmented proinflammatory cytokine response to lipopolysaccharide. Neuroimmunomodulation. 3:141-149.

31. Bhatia, M., et al. 1998. Role of substance $P$ and the neurokinin 1 receptor in acute pancreatitis and pancreatitis-associated lung injury. J. Clin. Invest. 95:4760-4765.

32. Castagliuolo, I., et al. 1998. Neurokinin-1 (NK-1) receptor is required in Clostridium difficile-induced enteritis. J. Clin. Invest. 101:1547-1550. 